\title{
Development of Novel Bacteriostatic Agents against Mycobacterium tuberculosis Using In silico Techniques
}

*Anupama Pandrangi

Osmania University, Hyderabad-500 007, India

\begin{abstract}
The cell wall of Mycobacterium tuberculosis is made up of mycolic acids which are found to play important role in pathogenesity by modification of double bonds at specific sites on mycolic acid precursors by the action of cyclopropane mycolic acid synthases (CMASs) that belong to a family of S-adenosyl-methionine-dependent methyl transferases. PcaA is an cyclopropane mycolic acid synthase required for cording, persistence and virulence of Mycobacterium tuberculosis and modifies mycolic acid by cyclopropanation of proximal double bond to cis cyclopropane generating alpha mycolic acid. A molecular docking of selected compounds was performed and the differences in their binding modes were investigated in order to design novel lead compound that can act as better antitubercular agent targeting cyclopropane mycolic acid synthases.
\end{abstract}

\section{Introduction}

Mycobacterium tuberculosis (Mtb) is an obligate human pathogen that causes tuberculosis (TB) and predominantly grows in the host phagocytes. The bacilli remain in a weakly acidic and noncytolytic environment by residing in the phagosomes of phagocytes and prevent the maturation and fusion of phagosomes with lysosomes $[1,2]$. Establishment of an all-around close apposition between the phagosome membrane and the mycobacterial surface known as phagosome maturation block (PMB) will occur only when phagosomes contain a single mycobacterium (loner phagosomes) [3]. However, when a phagosome contains more than one mycobacterium or mycobacterial clumps (social phagosome), $\mathrm{PMB}$ is not achieved leading to phagosome maturation and fusion with lysosomes [2, 3]. Thus, $\mathrm{PMB}$ is a strategy for altering the host immune response and there by sequestering pathogenic mycobacteria away from antigen presenting compartments [4].

Mycobacteria have an unusual cell wall whose outer layer is composed of mycolic acids which are very long-chain branched fatty acids that are either covalently attached to the cell wall or in the form of trehalose dimycolate (TDM), a toxic glycolipid of M. tuberculosis (Figure 1) [5]

Mycolic acids are produced by Claisen condensation between two fatty acyl chains i.e., long meromycoloyl chain (C40-C60) and a shorter saturated chain $(\mathrm{C} 22-\mathrm{C} 26)$ [6]. Various chemical modifications are introduced at proximal and distal positions of the meromycolic chain by a family of paralogous S-adenosylmethioninedependent methyltransferases (AdoMet-MTs), the mycolic acid methyltransferases (MA-MTs) generating three major mycolic acids: alpha mycolates (two cis cyclopropane rings), methoxymycolates (a single cis or trans cyclopropane ring and a methoxy group), and ketomycolates (a single cis or trans cyclopropane ring and a ketone group) (Figure 2) and these modifications are known to be important for the pathogenicity, virulence, and persistence of M. tuberculosis. Among $8 \mathrm{~S}$-adenosylmethionine (SAM)-dependent methyltransferases encoded by M. tuberculosis, 6 of them have been shown to participate in mycolic acid modification (MmaA1 to -4, PcaA, and CmaA2) [7-14].

Decoration of mycolic acids by cyclopropanation is a modification associated with pathogenic bacteria and have profound effects on the resistance of the mycobacteria to the oxidative stress [15], the fluidity and permeability of the cell wall $[10,16,17]$. Literature survey revealed that cyclopropane synthase is necessary for virulence and long-term persistence of pathogenic bacteria in vivo [14,19]. For example, cyclopropane synthase, PcaA which introduces the cis-cyclopropane at the proximal position of alpha-MAs has an impact on the persistence of the tubercle bacillus within infected organisms and mutational studies revealed that pcaA mutants were unable to persist within and kill infected mice, indicating that PcaA is necessary for the establishment of a lethal and chronic infection [14].

The cyclopropane synthase enzyme, PcaA is a mixture of alpha/ beta proteins. The core region consists of seven-stranded beta sheet with alpha helices flanking on either side. It has two binding sites which include cofactor SAM/SAH (Figure 3) binding site and acyl substrate binding site [18]. The cofactor SAM/SAH binding pocket is composed of four motifs among which motif I-III are highly conserved among all three SAM-methyltransferases i.e., CmaA1, CmaA2 and PcaA. Motif I binds the amino acid moiety and the ribose, motif II binds the ribose and adenine ring, and motif III binds the adenine ring of SAM/SAH. Amino acid residues in the acyl substrate binding site are exclusively hydrophobic.

In view of important role played by PcaA protein in persistence and virulence of mycobacteria, an attempt has been made to design novel lead inhibitors for PcaA protein using in silico techniques. Homology modeling of PcaA protein was performed using Modeller 9.14. Novel bacteriostatic compounds against PcaA protein of Mycobacterium tuberculosis reported in literature [19-21] were selected as ligands. Further, docking studies performed with the selected ligands revealed that the compound T-2 was found to possess highest potentiality in inhibition of cyclopropane synthase activity of PcaA protein. Based on

*Corresponding author: Anupama Pandrangi, Osmania University, Hyderabad-500 007, India, Tel: +91-9966193890; E-mail: p_anupama2002@yahoo.com

Received May 05, 2015; Accepted May 25, 2015; Published June 01, 2015

Citation: Pandrangi A (2015) Development of Novel Bacteriostatic Agents against Mycobacterium tuberculosis Using In silico Techniques. Pharm Anal Acta 6: 374. doi:10.4172/21532435.1000374

Copyright: ( 2015 Pandrangi A. This is an open-access article distributed under the terms of the Creative Commons Attribution License, which permits unrestricted use, distribution, and reproduction in any medium, provided the original author and source are credited. 


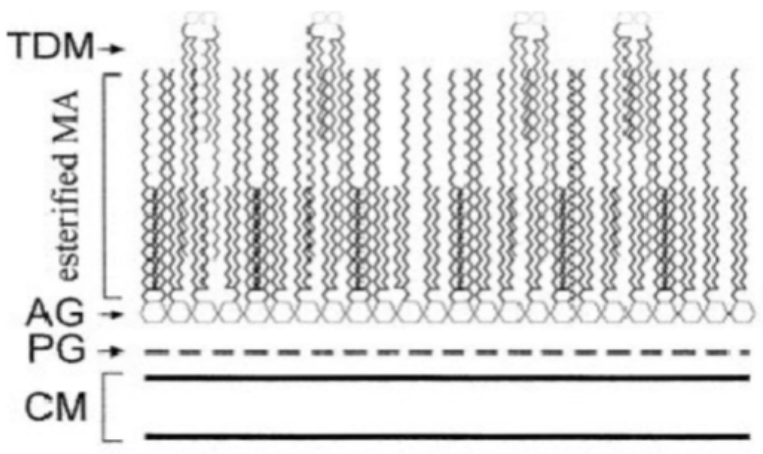

Figure 1: Schematic diagram of the cell envelope of $M$. tuberculosis (Glickman et al., 2000).

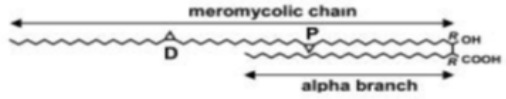

Type of Mycolate

Structure

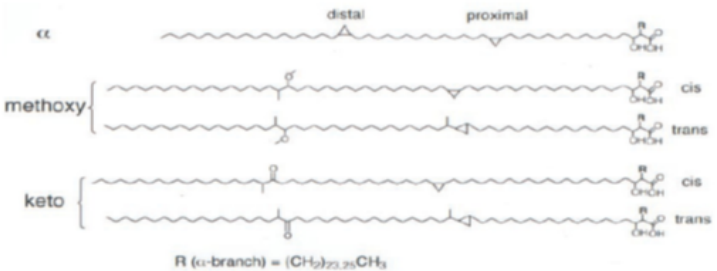

Figure 2: Structures of mycolic acids from M. tuberculosis (Julien Vaubourgeix et al., 2009 and Glickman et al., 2002). Alpha Mycolates contain cyclopropane rings at both the distal and the proximal positions. Methoxymycolates and ketomycolates have cis or trans cyclopropane rings at the proximal position and the oxygenated functional group at the distal position.

the scaffold of the ligand T-3, five new ligands F-1 to F-5 were designed and docking was performed.

\section{Materials and Methods}

\section{Sequence retrieval and template selection}

The amino acid sequence of PcaA protein is retrieved in FASTA format from SWISS PROT database followed by BLAST against PDB for template selection. With known structures available in the PDB, the BLAST is used to find the similarity of the sequence to closest homologous proteins and identifies the structure with high identity and similarity to be employed as template for homology modeling.

\section{Sequence alignment and model building}

The sequence alignment process is carried out by using ClustalW. 3D structure of PcaA is generated using "MODELLER 9.14". Energy minimization of the modeled structure is carried out by applying CHARMm force fields and steepest descent algorithm followed by conjugant gradient algorithm in DS until the convergence gradient is satisfied.

\section{Model validation}

RAMPAGE Server - Ramachandran plot analysis: Parameters like
Ramachandran plot quality, peptide bond planarity, bad nonbonded interactions, main chain hydrogen bond energy, C-alpha chirality and overall $\mathrm{G}$ factor, and the side chain parameters like standard deviations of chil gauche minus, trans and plus, and pooled standard deviations of chil with respect to refined structures were validated using RAMPAGE Server [22].

Prosa: This program $[23,24]$ compares $Z$ scores between target and template structure which are a measure of compatibility between its sequence and structure.

RMSD: Root Mean Squared Deviation (RMSD) is commonly used to represent the distance between two

objects and indicates the degree to which two three dimensional structures are similar. The lower the value is, the more similar the structures are. SPDBV program was used in calculating the RMSD value between the template $1 \mathrm{~L} 1 \mathrm{E}$ and our model structure.

\section{Molecular docking}

Ligand generation and optimization: All the compounds used for docking study were selected from the literature [19-21]. Ligand structures were constructed using ChemSketch Software (http://www. acdlabs.com). Catalyst algorithm in DS was used in ligand preparation with constraint parameters such as tautomer and isomer generation, removal of all the duplicate structures and generation of the $3 \mathrm{D}$ structure.

Docking studies: MOE program (Molecular Operating Environment) [25] designed by the Chemical Computing Group was used in performing docking and analysing the binding of the ligand molecules with the protein molecule. The parameters used for the Docking were, Total Runs $=50$, Cycle $/$ Runs $=15$, Iteration Limit $=10$ 000, Potential Energy Grid: ON, Annealing Algorithm: Simulated Annealing.

\section{Results and Discussion}

\section{Sequence retrieval and template selection}

The amino acid sequence of PcaA protein is taken from SWISSPROT database containing 287 residues with accession number: A2VFF5, entry name: A2VFF5_MYCTU, and protein name: Mycolic acid synthase PcaA (Cyclopropane synthase). The FASTA sequence of the protein (1-287) is retrieved and submitted to BLAST against PDB database. The BLAST results yield X-ray structure of 1L1E from Mycobacterium tuberculosis having the highest sequence identity of

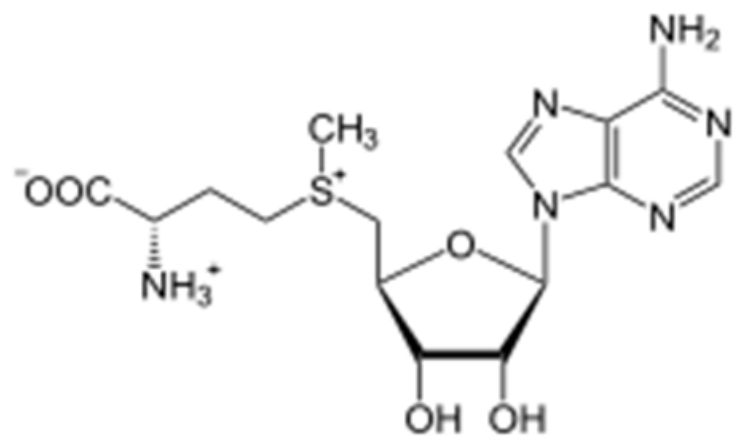

Figure 3: Structure of the co-factor SAM (S-Adenosyl methionine) 
$100 \%$ with a resolution of $2.0 \mathrm{~A} 0$. All the further procedures are carried out using MODELLER 9.14.

\section{Sequence alignment and model building}

The sequence of $1 \mathrm{~L} 1 \mathrm{E}$ is extracted and aligned with the PcaA protein of Mycobacterium tuberculosis using ClustalW. Based on this alignment as input, model of the PcaA is built using "MODELLER 9.14." Ten molecular models of PcaA are generated. The refinement process is carried out using DS by applying CHARMm force field and steepest descent method is applied with 0.001 minimizing RMS gradient and 2000 minimizing steps followed by conjugant gradient method till it reaches the satisfactory results for minimization. The best conformation of the model is shown in Figure 4.

\section{Model validation}

The quality of our model was evaluated by analyzing the final refined modeled structure of PcaA protein of Mycobacterium tuberculosis with RAMPAGE Server, Prosa, and RMSD calculation.

RAMPAGE Server - Ramachandran plot analysis: RAMPAGE Server revealed significant sterochemical quality in Ramachandran plot for the modeled target structure with favorable region (94.0\%), allowed region (3.9\%), and outlier region (2.1\%) respectively (Figure 5 and plot statistics are given in Table 1).

\section{Evaluation of residues}

Residue $[9: \mathrm{PHE}](179.42,165.12)$ in Allowed region

Residue [ 15 :HIS] $(-111.87,71.31)$ in Allowed region

Residue [ 16 :TYR] (-148.19,-127.66) in Allowed region

Residue [ 33 :TYR] $(-119.40,82.12)$ in Allowed region

Residue [ 176 :GLU] ( -49.76, -23.61) in Allowed region

Residue [ 178 :ARG] ( $-81.26,52.74)$ in Allowed region

Residue [ 181 :GLY] (-60.11, 102.99) in Allowed region

Residue [ 184 :LEU] $(-156.27,51.29)$ in Allowed region

Residue [ 185 :THR] ( -86.72, -97.68) in Allowed region

Residue [ 246 :ASN] $(-100.94,49.23)$ in Allowed region

Residue [ 254 :GLN] ( -89.91, -69.50) in Allowed region

Residue [ 177 :GLY] ( -50.02,-120.03) in Outlier region

Residue [ 179 :GLU] ( 169.75,-167.16) in Outlier region

Residue [ 180 :LYS] $(63.31,113.60)$ in Outlier region

Residue [ 200 :PHE] ( -89.97, 5.06) in Outlier region

Residue [ $201: \mathrm{PRO}](-31.50,118.27)$ in Outlier region

Residue [ 255 :SER] ( 156.97, 161.08) in Outlier region 94.0\%) $3.9 \%)$

Number of residues in allowed region $(\sim 2.0 \%$ expected $): 11$ (

Number of residues in outlier region $\quad: 6(2.1 \%)$

Prosa: Quality assessment of the model via Prosa revealed that the PcaA protein model matched NMR region of the plot with $\mathrm{Z}$ score
(-8.13) which is reliable to the $\mathrm{Z}$ score of the template 1L1E $(-8.0)$ (Figure 6). It signifies the quality of our model.

RMSD: The low RMSD between the target (PcaA protein) and template (1L1E) which was found to be $0.168 \mathrm{~A}^{\circ}$, reflects the presence of strong homology.

Active site identification: Using the tool panel "binding site," binding site analysis was performed. The receptor was analysed for the active sites from the "find sites from the receptor cavities," and Site 1 was selected from the displayed sites.

Docking studies: The binding modes of the selected ligands (Figure 7) in the active sites of modeled PcaA protein were studied using the software packageMOE program MOE (Molecular Operating Environment) [9] designed by the Chemical Computing Group. The pocket sequence of the active site was calculated using Site finder tool of MOE. The docking run generated 10 poses for each of the analogs and the dock scores are tabulated in Table 2. Analysis of the dock scores of all the selected ligands revealed that T-2 (Table 2) is having the highest dock score $-31.2429 \mathrm{Kcal} / \mathrm{mol}$. The crucial interaction of T-2 with PcaA protein is shown in Figure 8.

T-2 ligand is structurally similar to co-factor (Figure 1), except that the amino acid part of co-factor is replaced by 2 - $\mathrm{N}$-decylaminoethyl group. Literature survey revealed that the adenosine moiety of T-2 exactly replaces that of the cofactor, while the lipid chain is deeply buried in the hydrophobic environment provided by the residues lining the substrate-binding pocket. Thus, T-2 competes with both the cofactor and the substrate. Based on the scaffold of T-2, five new ligands F-1 to F-5 (Figure 9) were designed making slight variations in the side chain, and further docking was performed which revealed that ligand F-5 was found to possess highest dock score $-34.1412 \mathrm{Kcal} / \mathrm{mol}$. The dock sores of the newly designed ligands are tabulated in Table 3 and the binding interaction of the F-5 ligand with PcaA protein is shown in Figure 10.

\section{Conclusion}

In this study, we have predicted the structure for PcaA protein of Mycobacterium tuberculosis based on the template 1L1E. Ramchandran plot, Prosa and RMSD calculation between the target and template

\begin{tabular}{|c|c|c|c|}
\hline Structure & Favorable region & Allowed region & Outlier region \\
\hline PcaA & $94.0 \%$ & $3.9 \%$ & $2.1 \%$ \\
\hline
\end{tabular}

Table 1: The percentage of residues in the core region of the Ramachandran plot for the built PcaA model.

\begin{tabular}{|c|c|}
\hline Ligand & Dock Score $(\mathrm{Kcal} / \mathrm{mol})$ \\
\hline T-1 & -22.9240 \\
\hline T-2 & -31.2429 \\
\hline T-3 & -14.5829 \\
\hline
\end{tabular}

Table 2: Dock scores of the ligand compounds into the modeled PcaA protein active site.

\begin{tabular}{|c|c|}
\hline Llgand & Dock Score $(\mathrm{Kcal} / \mathrm{mol})$ \\
\hline F-1 & -27.0943 \\
\hline F-2 & -34.0764 \\
\hline F-3 & -29.0738 \\
\hline F-4 & -29.4136 \\
\hline F-5 & $-\mathbf{3 4 . 1 4 1 2}$ \\
\hline
\end{tabular}

Table 3: Dock scores of the ligand compounds into the modeled PcaA protein active site. 


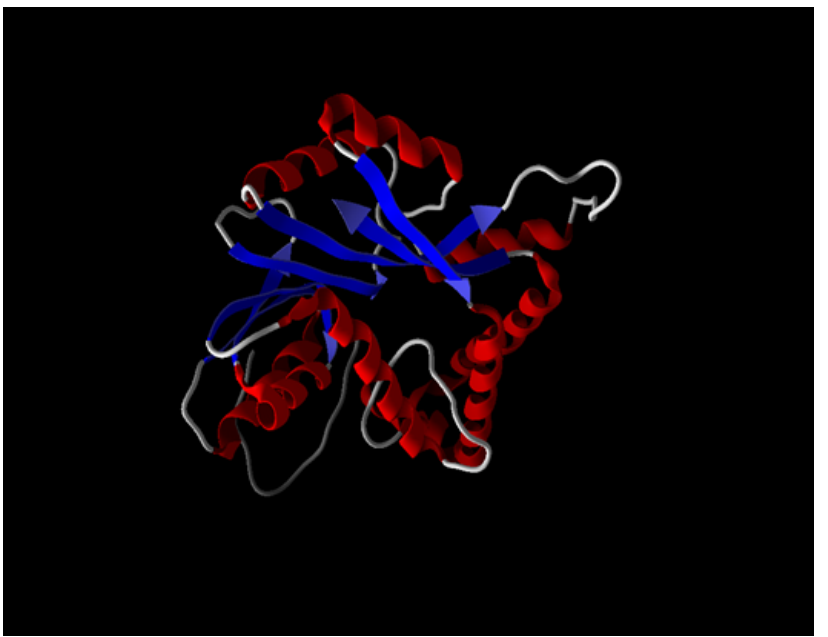

Figure 4: Modeled PcaA protein.

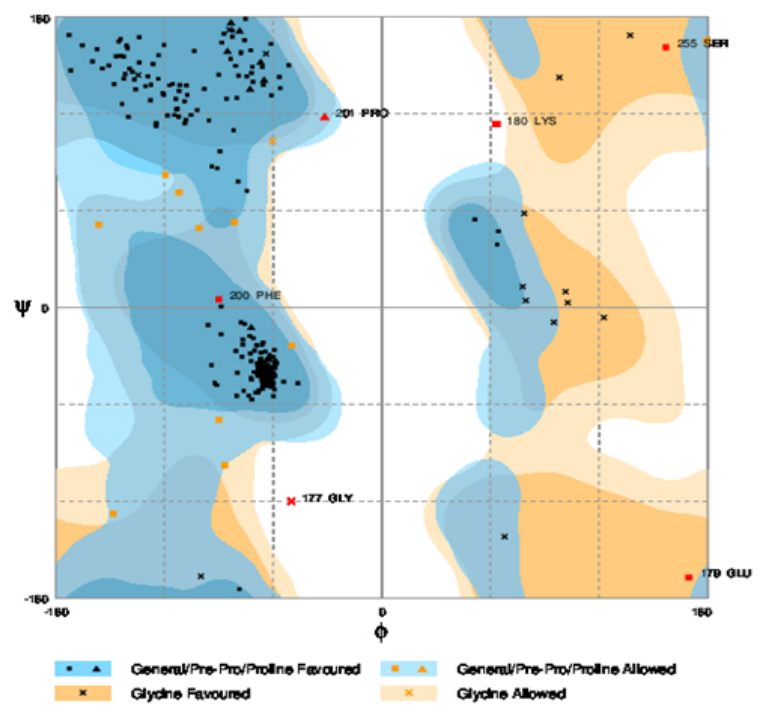

Figure 5: Ramachandran's Map of PcaA protein.
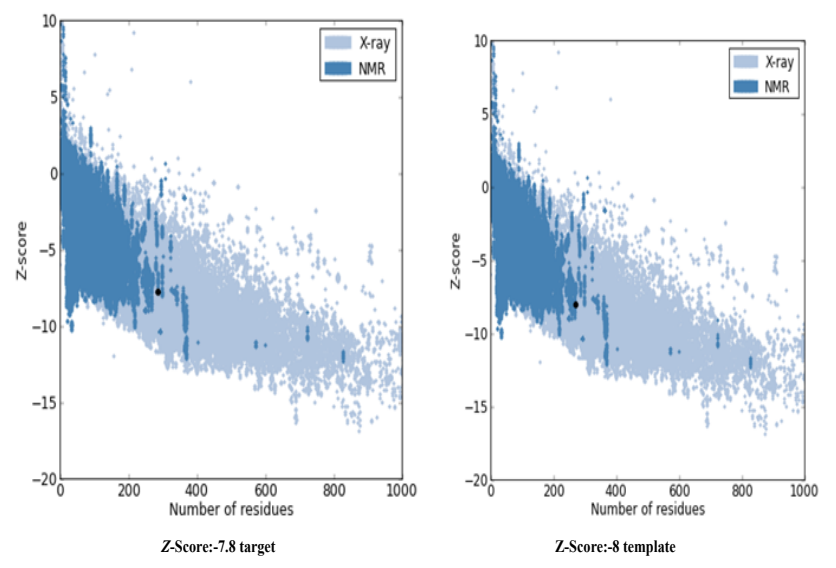

Figure 6: The plots of $Z$ Score values of target and template, determined by NMR (shown in dark blue color) and by X-ray (shown in light blue color) using Prosa program. The black dots point out the Z Scores of our model $(-7.81)$ and template $(-8.00)$.

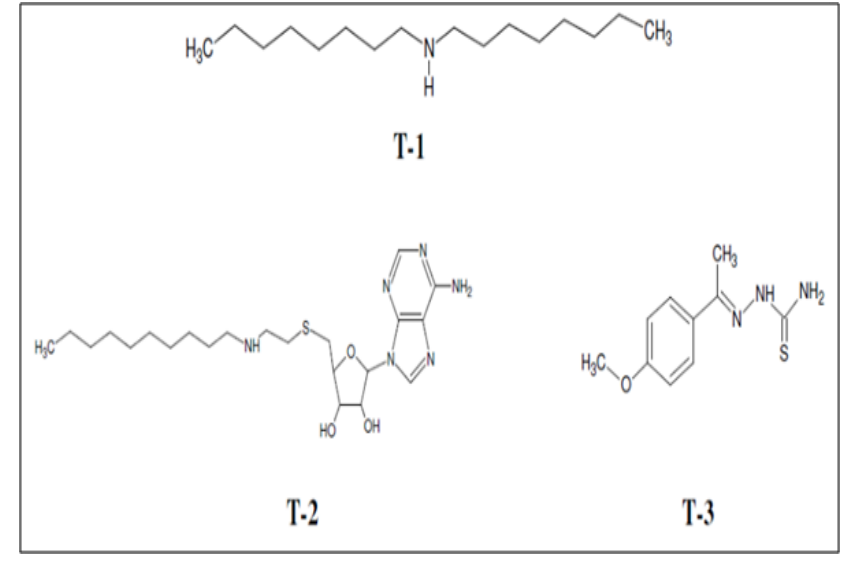

Figure 7: Ligands used for docking ( Ref 19-21).

(iiㅊ)

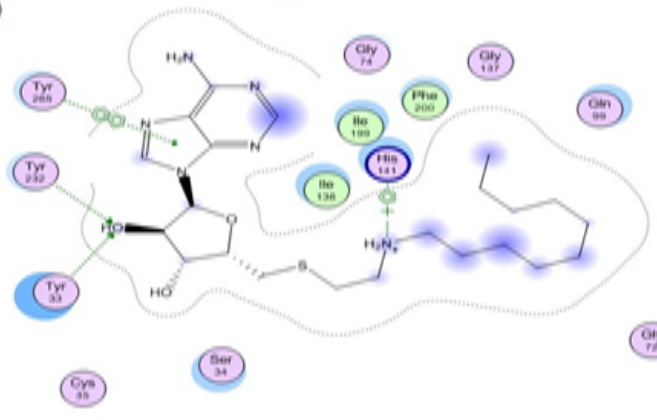

(iiv)

Figure 8: Binding interactions of T-2 ligand into the active site of protein.
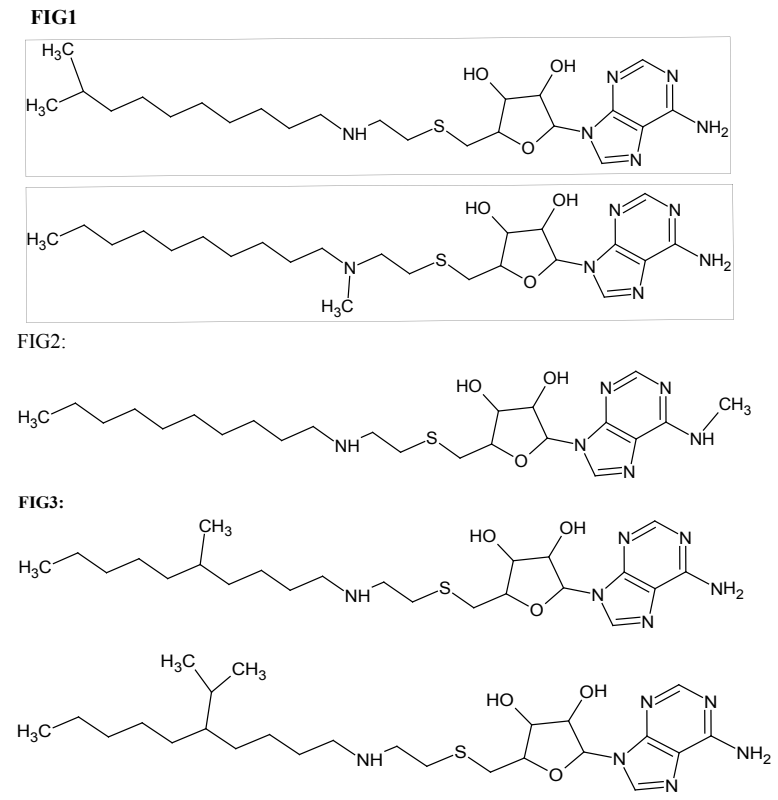

Figure 9: Newly designed ligands. 
Citation: Pandrangi A (2015) Development of Novel Bacteriostatic Agents against Mycobacterium tuberculosis Using In silico Techniques. Pharm Anal Acta 6: 374. doi:10.4172/21532435.1000374

Page 5 of 5

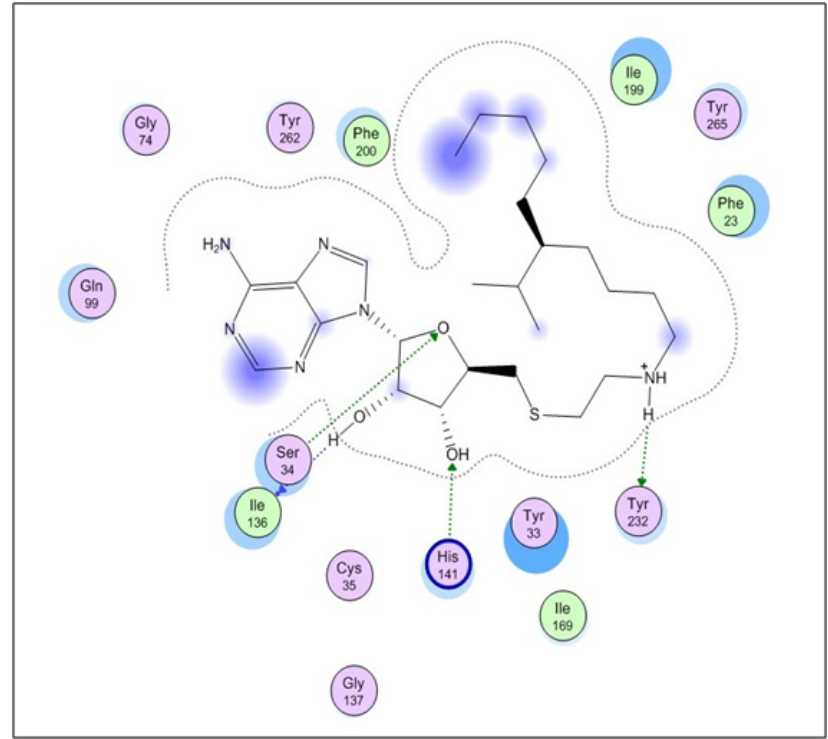

Figure 10: Binding interactions of F-5 ligand into the active site of protein.

proteins revealed the reliability of the model for docking. MOE was used to perform the docking studies. From the scoring functions and individual residue interactions we conclude that the compound T-2 has more affinity at active site than others. The T-2 ligand with a dock score of -31.2429

$\mathrm{Kcal} / \mathrm{mol}$ showed better results among the selected compounds from literature and has already been validated invitro as well as invivo. Further, based on the scaffold of T-2, five new ligands F-1 to F-5 were designed. Docking studies performed with the newly designed ligands reveal that F-5 with a dock score of $-34.1412 \mathrm{Kcal} / \mathrm{mol}$ could be good lead for development of novel bacteriostatic agents in inhibition of cyclopropane synthase activity of PcaA protein of Mycobacterium tuberculosis and has to be further validated in wet lab studies for its proper function.

\section{Conflict of Interests}

The author declares that there is no conflict of interests regarding the publication of this paper.

\section{Acknowledgment}

The author acknowledges Professor V. Uma, Department of Chemistry, Osmania University, for providing technical assistance in carrying out this work.

\section{References}

1. Armstrong JA, Hart PD (1971) "Response of cultured macrophages to Mycobacterium tuberculosis, with observations on fusion of lysosomes with phagosomes," J. Exp. Med 134: 713-740.

2. Chastellier C de, Forquet Gordon FA, Thilo L (2009) "Mycobacterium requires an all around closely apposing phagosome membrane to maintain the maturation block and this apposition is re-established when it rescues itself from phagolysosomes," Cell Microbiol 11: 1190-1207.

3. Chastellier $C$ de (2009) "The many niches and strategies used by pathogenic mycobacteria for survival within host macrophages," Immunobiology, 214: 526-542.

4. Flynn JL, Chan J (2003) "Immune evasion by Mycobacterium tuberculosis: living with the

5. enemy," Curr. Opin. Immunol. 15: 450-455.

6. 5. Barry CE III, et al. (1998) "Mycolic acids: structure, biosynthesis and physiological functions," Prog. Lipid Res., 37: 143-179.

7. Marrakchi H, Bardou F, Lanéelle MA, Daffé M (2008) The Mycobacterial Cell Envelope, American Society for Microbiology, Washington, DC, PP: 41-62.

8. Barkan D, Rao V, Sukenick GD, Glickman MS (2010) "Redundant function of cmaA2 and mmaA2 in Mycobacterium tuberculosis cis cyclopropanation of oxygenated mycolates" J Bacteriol 192: 3661-3668.

9. Behr MA, Schroeder BG, Brinkman JN, Slayden RA, Barry CE (2000) A point mutation in the mma3 gene is responsible for impaired methoxymycolic acid production in Mycobacterium bovis BCG strains obtained after 1927. J Bacterio 182: 3394-3399.

10. Dao DN, Sweeney K, Hsu T, Gurcha SS, Nascimento IP, et al (2008) Mycolic acid modification by the mmaA4 gene of $\mathrm{M}$. tuberculosis modulates $\mathrm{IL}-12$ production, PLoS Pathog 4: e1000081.

11. Dubnau E, Chan J, Raynaud C, Mohan VP, Lanéelle MA, et al. (2000) Oxygenated mycolic acids are necessary for virulence of Mycobacterium tuberculosis in mice, Mol Microbiol 36: 630-637.

12. Dubnau E, Marrakchi H, Smith I, Daffé M, Quémard A (1998) Mutations in the $\mathrm{cmaB}$ gene are responsible for the absence of methoxymycolic acid in Mycobacterium bovis BCG Pasteur Mol. Microbiol 29: 1526-1528.

13. Glickman MS (2003) The mmaA2 gene of Mycobacterium tuberculosis encodes the distal cyclopropane synthase of the alpha-mycolic acid, J Biol Chem 278 7844-7849.

14. Glickman MS, Cahill SM, Jacobs WR Jr (2001) The Mycobacterium tuberculosis cmaA2 gene encodes a mycolic acid trans-cyclopropane synthetase, J Biol Chem 276: 2228-2233.

15. Glickman MS, Cox JS, Jacobs WR Jr (2000) A novel mycolic acid cyclopropane synthetase is required for cording, persistence, and virulence of Mycobacterium tuberculosis, Mol Cell 5: 717-727.

16. Yuan Y, Lee RE, Besra GS, Belisle JT, Barry CE (1995) Identification of a gene involved in the biosynthesis of cyclopropanated mycolic acids in Mycobacterium tuberculosis" Proc. Natl. Acad. Sci. U. S. A 92: 6630-6634.

17. George KM, Yuan Y, Sherman DR, Barry CE $3^{\text {rd }}$ (1995) The Biosynthesis Of Cyclopropanated Mycolic Acids In Mycobacterium Tuberculosis. Identification And Functional Analysis Of CMAS-2, J Biol Chem 270: 27292-27298.

18. Yuan Y, Crane DC, Musser JM, Sreevatsan S, Barry CE 3rd (1997) MMAS-1, the Branch Point Between cis- and trans-Cyclopropane-containing Oxygenated Mycolates in Mycobacterium tuberculosis, J Biol Chem 272: 10041-10049.

19. Huang CC, Smith CV, Glickman MS, Jacobs WR Jr, Sacchettini JC (2002) Crystal Structures of Mycolic Acid Cyclopropane Synthases from Mycobacterium tuberculosis, J Biol Chem 277: 11559-11569.

20. Barkan D, Liu Z, Sacchettini JC, Glickman MS (2009) Mycolic acid cyclopropanation is essential for viability, drug resistance and cell wall integrity of Mycobacterium tuberculosis, Chem Biol 16: 499-509.

21. Vaubourgeix J, Bardou F, Boissier F, Julien S, Constant P, et al. (2009) S-Adenosyl-N-decyl-aminoethyl, a Potent Bisubstrate Inhibitor of Mycobacterium tuberculosis Mycolic Acid Methyltransferases, J Biol Chem 284: 19321-19330.

22. Alahari A, Trivelli X, Guérardel Y, Dover LG, Besra GS, et al. (2007) Thiacetazone, an Antitubercular Drug that Inhibits Cyclopropanation of Cell Wall Mycolic Acids in Mycobacteria, PLoS ONE 2: E1343.

23. Lovell SC, Davis IW, Arendall WB 3rd, de Bakker PI, Word JM (2002) Structure validation by Calpha geometry: phi,psi and Cbeta deviation. Proteins: Structure, Function \& Genetics 50: 437-450.

24. Wiederstein M, Sippl MJ (2007) ProSA-web: interactive web service for the recognition of errors in three-dimensional structures of proteins. Nucleic Acids Research 35: W407-W410.

25. Sippl MJ (1993) Recognition of Errors in Three-Dimensional Structures of Proteins. Proteins 17: 355-362.

26. MOE (The Molecular Operating Environment) version 2005.06, Chemical Computing Group, Inc. 